\title{
Omega-3 Fatty Acids for Autistic Spectrum Disorder: A Systematic Review
}

\author{
Stephen Bent $\cdot$ Kiah Bertoglio $\cdot$ Robert L. Hendren
}

Published online: 31 March 2009

(C) The Author(s) 2009. This article is published with open access at Springerlink.com

\begin{abstract}
We conducted a systematic review to determine the safety and efficacy of omega-3 fatty acids for autistic spectrum disorder (ASD). Articles were identified by a search of MEDLINE, EMBASE, and the Cochrane Database using the terms autism or autistic and omega-3 fatty acids. The search identified 143 potential articles and six satisfied all inclusion criteria. One small randomized controlled trial $(n=13)$ noted non-significant improvements in hyperactivity and stereotypy. The remaining five studies were small $(n=30,22,19,9$, and 1) with four reporting improvements in a wide range of outcomes including language and learning skills, parental observations of general health and behavior, a clinicianadministered symptom scale, and clinical observations of anxiety. Due to the limitations of evidence from uncontrolled studies and the presence of only one small randomized controlled trial, there is currently insufficient scientific evidence to determine if omega-3 fatty acids are safe or effective for ASD.
\end{abstract}

Keywords Autism - Omega-3 fatty acids . Complementary and alternative medicine

S. Bent $(\bowtie)$

Department of Medicine, Osher Center for Integrative Medicine, University of California, San Francisco VAMC,

111-A1 4150 Clement Street, San Francisco, CA 94121, USA

e-mail: Stephen.Bent@ucsf.edu

S. Bent

Department of Epidemiology and Biostatistics,

University of California, San Francisco, CA, USA

K. Bertoglio · R. L. Hendren

Department of Psychiatry, MIND Institute,

University of California, Davis, Sacramento, CA, USA

\section{Introduction}

Complementary and alternative medical (CAM) therapies are commonly used by patients with autistic spectrum disorders (ASDs). In four recent surveys, the prevalence of CAM use was 32, 52, 74, and 95\% (Hanson et al. 2007; Harrington et al. 2006; Levy et al. 2003; Wong and Smith 2006). The variability in the reported prevalence is likely related to the substantial differences in survey designs and the populations studied, but it is clear that CAM use is common.

Omega-3 fatty acids are among the most commonly used CAM therapies, and have been reported to be currently used by $28.7 \%$ of children with ASDs (Green et al. 2006). Omega-3 fatty acids are polyunsaturated fatty acids and three main types are found in the human diet: ALA (alpha-linolenic acid), DHA (docosahexaenoic acid), and EPA (eicosapentaenoic acid). DHA and EPA are found in seafood, while ALA is found in nut and plant oils. Interestingly, fish do not produce EPA and DHA, but the oils are synthesized by single-cell marine organisms that are eaten by fish (Harris 2004). While the human body can synthesize both DHA and EPA from ALA, it can not synthesize any of these three types of fatty acids "from scratch." Thus, these substances are typically considered essential human nutrients and are often called "essential fatty acids" (Freeman et al. 2006). DHA and EPA are used by the body to produce a variety of different compounds, including cyclooxygenases and lipoxygenases, which have many different physiological actions (Harris 2004).

While the potential mechanism of action of omega-3 fatty acids for improving symptoms of ASD is unknown, neural tissue contains high concentrations of DHA, and studies suggest that this fatty acid is essential to the growth and functional development of the human brain (Freeman 
et al. 2006). Omega-3 fatty acids are also known to exert an anti-inflammatory effect (Kris-Etherton et al. 2002). Three prior studies have reported low levels of omega- 3 fatty acids in children with ASD compared to controls (Bell et al. 2004; Meguid et al. 2008; Vancassel et al. 2001), while a fourth found no difference (Bu et al. 2006). Fatty acid deficiencies have also been reported in individuals with other psychiatric disorders, including schizophrenia and attention deficit hyperactivity disorder. A recent systematic review found statistically significant benefits from omega-3 fatty acids for the treatment of depression, although there was considerable heterogeneity in the results and marked variation in the methodology of included studies (Freeman et al. 2006).

Because omega- 3 fatty acids are commonly used by children with ASDs and there is some limited evidence regarding a possible physiological basis for beneficial effects, we conducted a systematic review to identify and evaluate all prior clinical studies that reported treatment effects of omega-3 fatty acids in children with ASDs.

\section{Methods}

\section{Protocol}

All study procedures were defined a priori in a study protocol that specified the search strategy, the inclusion and exclusion criteria, the quality rating system, and the method of analysis.

\section{Search}

We searched MEDLINE, EMBASE, and the Cochrane Collaboration Clinical Trials Registry from 1966 to September 2008 to identify relevant studies in all languages. The search strategy used the following terms: (autism OR autistic) AND (unsaturated fatty acid OR unsaturated fatty acids OR omega 3 OR omega3). We reviewed the reference lists of all identified studies and contacted experts to identify additional studies.

\section{Study Selection}

We set broad inclusion criteria to include not only randomized controlled trials but also any other study design that (1) enrolled human subjects of any age who had ASDs, (2) treated patients with omega-3 fatty acids with any dose and duration, and (3) reported at least one outcome measure (including clinical or parental observation) that addresses the core symptoms of ASDs (social difficulties, communication problems, and repetitive or restrictive behaviors) or any associated symptom (such as sleep disturbance, gastrointestinal problems, or anxiety). We excluded studies that combined omega- 3 fatty acids with other interventions. The primary reason for setting broad inclusion criteria was to evaluate not only the randomized controlled trials, but also other study designs that may be commonly presented to families and care providers as the rationale to begin treatment with omega-3 fatty acids. Our goal was to evaluate all studies in an unbiased manner to summarize the current scientific evidence for families and clinicians who are making treatment decisions.

\section{Quality Assessment and Data Abstraction}

For each study, two authors independently abstracted data regarding study eligibility, study quality, treatment, and ASD-related outcomes. For randomized controlled trials, study quality was assessed using the Jadad scale (Jadad et al. 1996). For designs other than randomized controlled trials, we provided a written description of the design and the potential limitations, but did not provide a quantitative quality score, as there is no widely accepted tool for other designs.

\section{Clinical Recommendation}

In order to provide a concise clinical recommendation about whether the use of omega-3 fatty acids is indicated based on a review of current scientific evidence, we chose to follow the methods of the US Preventive Services Task Force (USPSTF), which provides letter grades evaluating the certainty and magnitude of the benefit of an intervention (letters A-D and "I" for insufficient information; Sawaya et al. 2007). In general, letter grades of A or B indicate that the treatment should be provided to eligible patients, treatments with a letter grade of $\mathrm{C}$ should not be offered routinely, and D-grade treatments should not be provided. Treatments with insufficient evidence are rated as "I," and no recommendation is made regarding clinical use (Sawaya et al. 2007).Two authors (SB and KB) independently followed the methodology of this rating framework to determine the clinical recommendation.

\section{Results}

The database searches, reviews of bibliographies, and contact with experts yielded 143 potentially relevant articles. Six articles satisfied all inclusion and no exclusion criteria and are included in this systematic review. Reasons for exclusion of other articles are shown in Fig. 1.

The characteristics of the six included studies are shown in Table 1. 


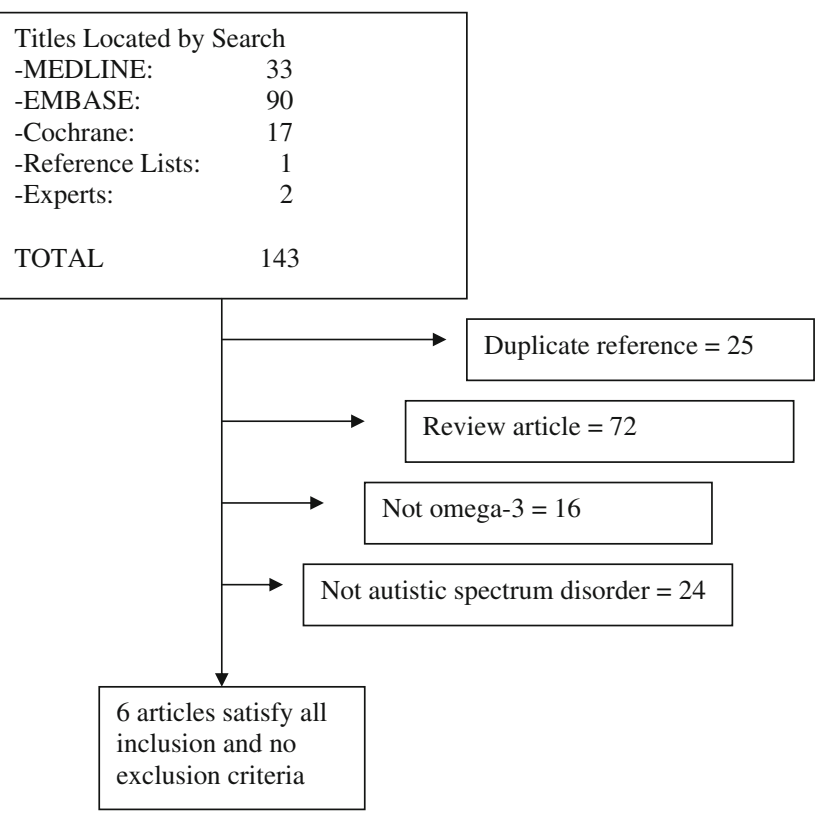

Fig. 1 Reason for exclusion of studies

Only one study was a randomized controlled trial (Amminger et al. 2007). This study enrolled 13 children with autism (based on DSM-IV criteria and the ADI-R) and randomly assigned them to the daily use of $1.5 \mathrm{gms}$ of omega-3 fatty acids or an identical placebo for 6 weeks. The outcome measure was the Aberrant Behavior Checklist. The difference in the change in each of the subscales between groups is shown in Table 2 .

Each subscale showed a greater improvement in the omega-3 group compared to the placebo group, but none of these changes reached statistical significance. The largest changes were in the hyperactivity and stereotypy subscales. The study was methodologically sound, and received a four out of five point rating on the Jadad score (the score was reduced by one point because the method of randomization was not described).

Four studies were uncontrolled, open-label studies that enrolled children or young adults with autism or Asperger's. Politi et al. 2008 conducted an open-label study of 19 young adults (mean age 29) with severe autism, moderate to profound mental retardation, and severe maladaptive behaviors. All subjects were given 0.93 gms of omega-3 fatty acids (DHA + EPA) and a vitamin supplement containing $5 \mathrm{mg}$ of vitamin $\mathrm{E}$ daily for 6 weeks. The frequency and severity of problematic behaviors was assessed using an instrument (the Rossago Behavioral Checklist) for 6 weeks before, during, and after treatment (18 week total study period). The authors found no improvement in the mean severity score of problematic behaviors between the pre-treatment and treatment periods. Interestingly, there appeared to be an improvement in both the frequency and severity of symptoms in the post-treatment period, though it is not clear if this was due to beneficial effects of omega3 fatty acids or other factors (as there was no control group).

Meguid et al. 2008 treated 30 children with autism from a National Research Center in Egypt for 3 months with a combination of omega-3 (240 mg DHA + $52 \mathrm{mg}$ EPA daily) and omega- 6 fatty acids $(68 \mathrm{mg})$ and Vitamin E. They reported that 20 of 30 children improved on the Childhood Autism Rating Scale, but they did not report the mean change in the overall group of 30 children.

Patrick et al. enrolled 22 children who were all treated in an open-label manner with one daily capsule containing $247 \mathrm{mg}$ per day of omega-3 fatty acids for 90 days (Patrick and Salik 2005). The authors reported that there was a statistically significant increase from day 0 to 90 in each of the subscales of the assessment of basic language and learning skills. However, no raw data were presented.

Bell et al. 2004 included a very brief description of an open-label study where nine children with autism or Asperger's were given one of two different omega-3 supplements of varying dose for at least 6 months. No structured outcomes were assessed, but parents reported improvements in general health and a variety of outcome measures (Table 2).

The sixth study was a case report involving an 11 yearold child who had been diagnosed with autism at age 2.5 and was having problems with high levels of anxiety and agitation associated with compulsive rituals (Johnson and Hollander 2003). Fish oils were initiated and advanced to $3 \mathrm{~g} /$ day (540 mg EPA). The parents and the clinician reported complete elimination of anxiety and agitation after 1 week, and the improvement was stable over 8 months of follow-up.

Only the randomized controlled trial reported the details of the method of ascertaining adverse medication effects (UKU Side Effect Rating Scale) (Amminger et al. 2007). In this study, one child withdrew due to gastrointestinal complaints and lack of perceived benefit. The authors noted that a mild adverse event of fever was reported in the omega-3 group (but the number of patients reporting this was not shown). In one uncontrolled study, 2/22 children withdrew due to reports of increased physical activity, but no other adverse effects were noted (Patrick and Salik 2005). In another uncontrolled study, a "few parents" reported "increased hyperactivity and behavioral problems" (Bell et al. 2004). Two uncontrolled studies (Meguid et al. 2008; Politi et al. 2008) and the case report (Johnson and Hollander 2003) did not discuss whether adverse events were assessed.

Based on the evidence summarized above, two independent raters agreed that the evidence for efficacy of omega-3 fatty acids for the treatment of autism should be 


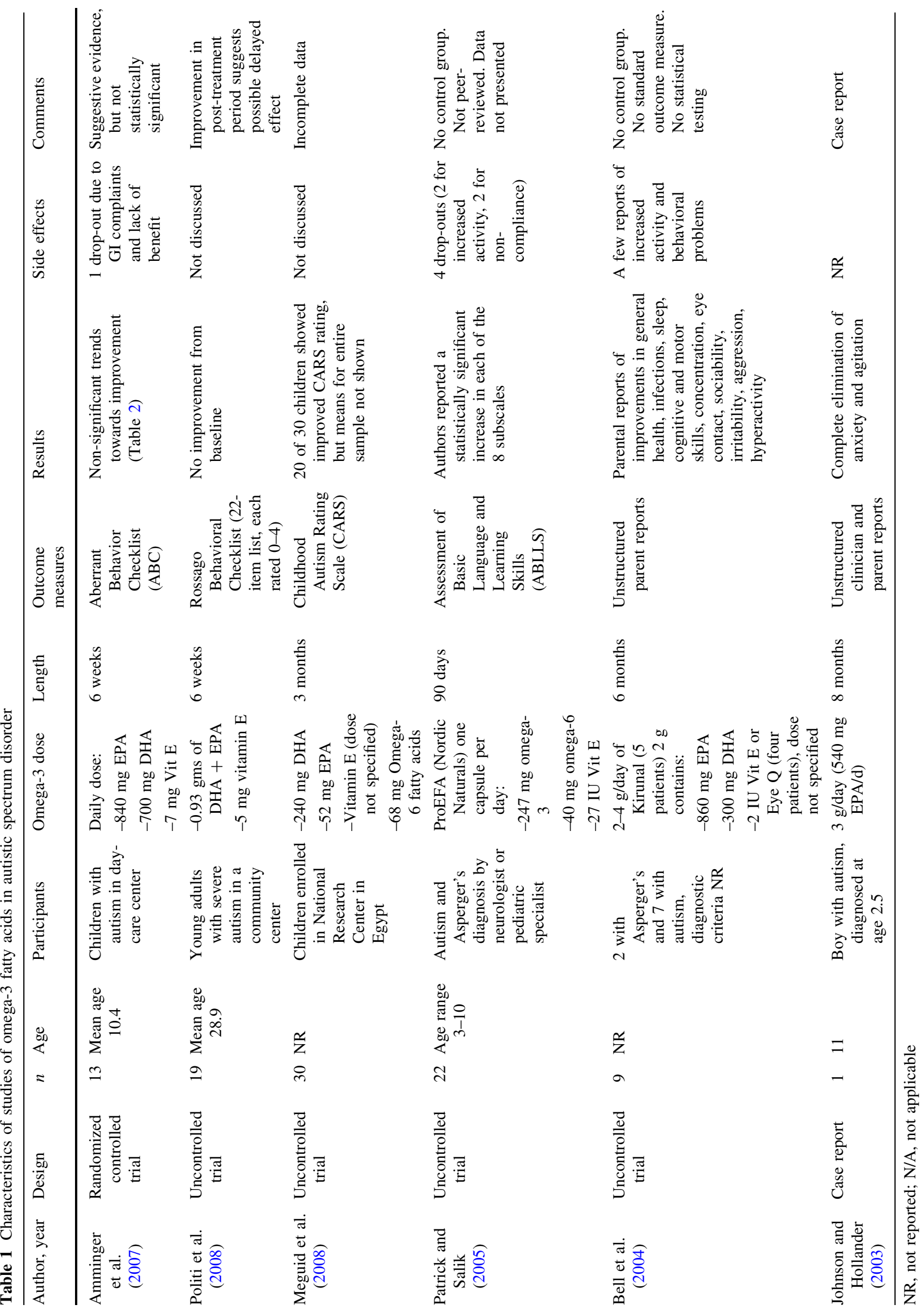


Table 2 Results of the one randomized controlled trial of omega-3 fatty acids (Amminger et al. 2007)

\begin{tabular}{|c|c|c|c|c|c|c|}
\hline $\begin{array}{l}\text { Sub-scale of the aberrant } \\
\text { behavior checklist }\end{array}$ & Baseline (SD) ${ }^{\mathrm{a}}$ & $\begin{array}{l}\text { Post-treatment }(\mathrm{SD}) \\
6 \text { weeks }\end{array}$ & $\begin{array}{l}\text { Change in } \\
\text { Score (SD) }\end{array}$ & $\begin{array}{l}\text { Difference in change: } \\
\text { active }- \text { control }^{\mathrm{b}}\end{array}$ & $\begin{array}{l}95 \% \text { confidence } \\
\text { interval }\end{array}$ & Effect size \\
\hline Irritability & $\begin{array}{l}\text { A: } 29.3(9.2) \\
\text { P: } 26.4(5.7)\end{array}$ & $\begin{array}{l}\text { A: } 24.6(8.7) \\
\text { P: } 21.8(2.8)\end{array}$ & $\begin{array}{l}\text { A: } 4.7(3.5) \\
\text { P: } 4.6(7.5)\end{array}$ & 0.1 & $(-9.0,9.2)$ & 0.02 \\
\hline Social withdrawal & $\begin{array}{l}\text { A: } 24.4(12.0) \\
\text { P: } 25.6(4.4)\end{array}$ & $\begin{array}{l}\text { A: } 18.9(13.3) \\
\text { P: } 21.0(2.0)\end{array}$ & $\begin{array}{l}\text { A: } 5.6(8.1) \\
\text { P: } 4.6(5.6)\end{array}$ & 1.0 & $(-7.8,9.8)$ & 0.17 \\
\hline Stereotypy & $\begin{array}{l}\text { A: } 14.4(5.1) \\
\text { P: } 7.8(6.4)\end{array}$ & $\begin{array}{l}\text { A: } 13.0(5.2) \\
\text { P: } 8.8(4.1)\end{array}$ & $\begin{array}{l}\text { A: } 1.4(2.2) \\
\text { P: }-1.0(3.4)\end{array}$ & 2.4 & $(-1.8,6.6)$ & 0.72 \\
\hline Hyperactivity & $\begin{array}{l}\text { A: } 33.3(4.8) \\
\text { P: } 24.6(5.5)\end{array}$ & $\begin{array}{l}\text { A: } 29.3(5.7) \\
\text { P: } 27.6(5.9)\end{array}$ & $\begin{array}{l}\text { A: } 4.0(2.4) \\
\text { P: }-3.0(9.9)\end{array}$ & 7.0 & $(-5.2,19.2)$ & 0.71 \\
\hline Inappropriate speech & $\begin{array}{l}\text { A: } 8.3(4.0) \\
\text { P: } 9.0(1.6)\end{array}$ & $\begin{array}{l}\text { A: } 7.6(4.0) \\
\text { P: } 9.4(2.9)\end{array}$ & $\begin{array}{l}\text { A: } 0.7(3.0) \\
\text { P: }-0.4(2.9)\end{array}$ & 1.1 & $(-2.8,5.0)$ & 0.39 \\
\hline
\end{tabular}

a "A" indicates scores in the active (omega-3) group, and "P" indicates scores in the placebo group

b Positive values indicate that the treatment group had a greater benefit (change in the measure over the 6 week study period) than the placebo group. The confidence intervals surrounding this difference were generated and were not presented in the original paper. Confidence intervals do not assume equal variances; $t$-statistics computed using Satterhwaite's degrees of freedom

rated as "I," indicating that there is insufficient evidence to determine if it is effective.

\section{Discussion}

Despite the high prevalence of use of omega- 3 fatty acids among children with ASD's, there is very limited scientific evidence evaluating the safety and efficacy of the supplement in this population. We conducted an extensive literature search in all languages and found only one randomized controlled trial. The randomized controlled trial reported a small, non-significant trend towards benefit in the hyperactivity and stereotypy subscales of the Aberrant Behavior Checklist. While this pilot study was methodologically sound, it was limited by a small sample size and short duration, and it did not examine outcomes other than aberrant behavior. Also, because the omega-3 group had higher (or "worse") scores for hyperactivity and stereotypy at baseline than the placebo group, the observed benefits may have been due to regression to the mean, with the more severe initial measurements (in the omega-3 group) improving more through natural variation than the less severe initial scores (in the placebo group; Gilbert 2008).

The four uncontrolled studies (Bell et al. 2004; Meguid et al. 2008; Patrick and Salik 2005; Politi et al. 2008) and the one case report (Johnson and Hollander 2003) provide interesting, hypothesis-generating information and raise the possibility that omega- 3 fatty acids may have some benefits. However, these studies are significantly limited by the lack of a control group, and are therefore not able to determine if the reported improvements are due to omega-3 fatty acids or to the natural variation in symptoms of ASD that may be due to many other factors. Similarly, the lack of blinding makes all of the uncontrolled studies susceptible to reporting bias. Given the limitations of current studies on omega-3 fatty acids for ASD, families should be educated to properly weigh the evidence when considering treatment decisions. A plain-language summary of this article is provided in Appendix 1. A plain-language summary of the key issues related to interpreting scientific evidence in studies of CAM in ASD is provided in Appendix 2.

Prior studies examining the prevalence of omega-3 fatty acid deficiencies in children with ASD have found inconsistent results. One case-control study found that children with classic autism or Asperger's had a 10\% lower level of total omega-3 fatty acids compared to controls (Bell et al. 2004), and a second case-control study found a $20 \%$ lower level of omega-3 fatty acids (Vancassel et al. 2001). A third case-control study found no difference in omega-3 fatty acids levels when comparing 40 children with autism to 20 children with other developmental disabilities or 20 typically developing children (Bu et al. 2006). The reasons for these inconsistent findings are unclear, but may relate to differences in the selection of control populations or differences in laboratory methods of measuring fatty acids. If omega-3 fatty acids have beneficial effects, it is possible that these effects may be limited to a subset of children with ASD. Future studies should consider measuring omega-3 fatty acid levels over the course of a clinical trial to determine if any beneficial effects are limited to children with initial deficiencies or specific fatty acid profiles.

In summary, this systematic review identified one randomized controlled trial, four uncontrolled studies, and one case report examining the efficacy of omega-3 fatty acids 
for the treatment of ASD. Overall, there is insufficient scientific evidence to determine if omega- 3 fatty acids are beneficial for symptoms of ASD (Evidence Rating = "I" or Insufficient). Although there is very limited evidence regarding the efficacy of this therapy, future studies are indicated based on the high prevalence of use, the favorable initial safety profile and low cost, and the lack of studies with sufficient size to identify clinically important benefits. Since the one randomized controlled trial found the largest trend suggesting a possible benefit for improving hyperactivity, future studies should target this symptom area as the primary outcome measure. Measurement of free fatty acid levels during the course of future studies has the potential to identify subsets of patients who might benefit from this therapy. Future studies would also benefit from larger sample sizes with sufficient power to detect clinically important benefits, a longer duration to examine the time-course of treatment effects, a careful assessment of side effects and safety, and a determination of the adequacy of blinding. These recommendations are consistent with the widely accepted guidelines for the conduct and reporting of clinical trials (CONSORT Statement; Altman et al. 2001).

Acknowledgments This work was supported by grants from Autism Speaks, the Higgins Family Foundation, The Hellman Family Foundation, and the Emch Foundation (Dr. Bent).

Open Access This article is distributed under the terms of the Creative Commons Attribution Noncommercial License which permits any noncommercial use, distribution, and reproduction in any medium, provided the original author(s) and source are credited.

\section{Appendix 1: Patient Information Handout: Safety and Efficacy of Omega-3 Fatty Acids for Autistic Spectrum Disorders (ASD)}

\section{Background}

Omega-3 fatty acids are among the most commonly used CAM therapies in children with ASDs. These fatty acids are often called "essential" fatty acids, because they cannot be made in the human body and therefore must be consumed in the diet. Two types of omega- 3 fatty acids come from fish (DHA and EPA), while another comes from nut and plant oils (ALA). In the body, these fatty acids are used to produce hormone-like substances that are involved in a wide array of functions. Also, brain and nerve tissues contain high concentrations of DHA, and studies suggest that this fatty acid is essential to the growth of the nervous system.

There is some preliminary evidence that fatty acid levels may be low in certain psychiatric disorders, including schizophrenia and attention deficit hyperactivity disorder.
Studies of DHA and EPA suggest that these substances may be beneficial in the treatment of depression (Freeman et al. 2006). Three studies have found that omega- 3 fatty acid levels were low in children with autism (Bell et al. 2004; Meguid et al. 2008; Vancassel et al. 2001), while a fourth did not (Bu et al. 2006). Some have theorized that, since omega-3 fatty acid levels may be low in children with autism, supplementation might lead to an improvement in symptoms.

Are Omega-3 Fatty Acids Effective for Symptoms of ASD?

As with most CAM therapies for ASD, there is very little scientific evidence regarding the efficacy of omega-3 fatty acids. Two case series (which are reports that describe a group of children who took omega-3 fatty acids, but without a comparison to a control group) describe a number of benefits in language and learning skills, social skills, and other measures of health (Bell et al. 2004; Patrick and Salik 2005). One case series in young adults with severe autism reported no benefit after 6 weeks of treatment (Politi et al. 2008), and another reported benefits in a subset of patients (Meguid et al. 2008). One small, randomized, placebo-controlled trial in 13 children with ASD found a suggestion of a benefit in hyperactivity (Amminger et al. 2007). An ongoing study is further examining the potential benefits.

\section{Are Omega-3 Fatty Acids Safe?}

Omega-3 fatty acids are commonly used in adults and are now recommended as a standard therapy for the prevention of heart disease in adults with known heart disease. Most studies indicate that omega-3 fatty acids are relatively safe, although there are some concerns that it may increase the risk of bleeding (and therefore should be avoided in persons at increased risk for bleeding).

\section{Bottom Line}

There is insufficient scientific evidence to determine if omega-3 fatty acids are beneficial for ASDs. This supplement is believed to be relatively safe. It is always recommended that you discuss the potential risks and benefits of this and any other therapy with your child's regular health care provider.

Selection of Products

Omega-3 fatty acid products are considered to be "dietary supplements" and therefore have limited regulation and oversight from the US Food and Drug Administration 
(FDA). Dietary supplement products may have variability in quality, consistency, and safety testing. Because omega3 fatty acids come from fish, and some fish contain mercury, there is a concern that omega-3 fatty acid supplements could contain unsafe levels of mercury. Products should be tested to ensure mercury is not present. The FDA has advised that adults can safely consume a total of 3 grams per day of combined DHA and EPA, with no more than $2 \mathrm{~g}$ per day coming from dietary supplements ("FDA announces qualified health claims for omega-3 fatty acids," 2004). Dosing guidelines are not available for children. Studies of omega-3 fatty acid in children with autism have used daily supplement doses ranging from 0.247 to 1.54 grams of EPA plus DHA per day, but the size and duration of these studies is insufficient to determine the safety of these doses. If you and your health care provider decide to use omega-3 fatty acids with your child, we recommend the following resource to help in the selection of specific products:

Consumerlab.com - is a Web-based information service (subscription required) that analyzes the content of dietary supplements to determine if the label correctly reports the actual ingredients and whether the products meet current accepted standards for contents of specific products (http://www.consumerlab.com).

\section{Appendix 2: Patient Handout-Complementary and Alternative Medicine (CAM) for Autistic Spectrum Disorders (ASD)}

\section{What is Complementary and Alternative Medicine?}

Complementary and Alternative Medicine (CAM) is variably defined but generally refers to medical therapies and practices that are not commonly taught at medical schools or available at major hospitals and clinics in the United States (Eisenberg et al. 1998, 1993; in contrast to "traditional" or "conventional" medicine, which refers to therapies that are provided in most hospitals and clinics).

Sometimes complementary medicine is described as non-traditional therapies that are used in conjunction with traditional therapies (to complement their action), while alternative medicine is used to describe non-traditional therapies that are used in place of (as an alternative to) traditional therapies. Practically speaking, most non-traditional therapies are simply classified under the general term: CAM therapies. A related term used in some settings is integrative medicine, which highlights the belief that CAM therapies are best used when integrated with (rather than replacing) conventional medical care.
What are the major types of CAM?

There are many different ways to categorize CAM therapies. The National Center for Complementary and Alternative Medicine (NCCAM-see: http://nccam.nih. gov/) is the scientific branch of the National Institutes of Health that provides research funding for CAM. It outlines four "domains" of CAM:

1. Mind-Body Medicine: techniques used to "enhance the mind's capacity to affect bodily functions and symptoms," including meditation, prayer, mental healing, and therapies that use creative outlets such as art, music, and dance.

2. Biologically-Based Practices: products found in nature, such as herbs and vitamins, which may act similarly to drugs by affecting some biological pathway.

3. Manipulative and Body-Based Practices: such as chiropractic and massage that involve the manipulation of body parts.

4. Energy Medicine: practices that are designed to affect proposed "energy fields" surrounding the body (examples include qi gong, Reiki, and Therapeutic touch), as well as the use of conventional electromagnetic fields to affect diseases or symptoms.

Are CAM Therapies Used by Families of Children with ASDs?

CAM therapies are commonly used by families in treating their children affected by ASDs. In four recent surveys, the prevalence of CAM use was 32, 52, 74, and 95\% (Hanson et al. 2007; Harrington et al. 2006; Levy et al. 2003; Wong and Smith 2006). The variability in the prevalence of use is likely related to differences in survey design and the groups of children studied, but it is clear that CAM use is common.

Which CAM Therapies are Most Commonly Used for ASDs?

It is difficult to determine which CAM therapies are most commonly used, as most prior surveys have been limited to small or selected groups of patients.

Why do Families Use CAM Therapies for ASDs?

There is very little information available that examines how and why families decide to use CAM therapies for ASD. One study found that parents had a variety of goals in mind, including improving the general symptoms of ASD 
(social difficulties, communication, repetitive or restricted behaviors) as well as treating associated problems such as gastrointestinal problems, sleep problems, or to maintain general health (Wong and Smith 2006).

\section{How do I Decide Whether To Try a CAM Therapy for My Child Who Has an ASD?}

As with any medical therapy, deciding whether to use a specific CAM therapy involves a review of scientific evidence, focusing on the potential RISKS and BENEFITS of any therapy. Some general guidelines in evaluating the risks and benefits of each therapy are:

1. Risk-Benefit Analysis: Therapies that have a very low risk (low chance of side effects) and a very high chance of benefit have the greatest chance of being helpful for your child.

2. Limited current information for most CAM therapies: Unfortunately, for most CAM therapies, there is currently very limited information regarding potential risks and benefits. In the absence of scientific evidence, it may be fairly obvious that some therapies (such as art therapy) are safe, while it may be more difficult to determine the safety of others (such as high-dose vitamins).

3. Discuss CAM therapies with your care providers: Some families feel uncomfortable discussing their thoughts about using CAM therapies. However, health care providers understand that CAM use is very common and they want to be helpful in the difficult process of deciding whether to use specific therapies. Think of your care provider as your "coach," who will help you evaluate the evidence and alternatives- but ultimately, you will decide what is best for your child. Also, when using a CAM therapy, it is much safer to have a health care provider help monitor for possible side effects and benefits.

4. Delays in the use of proven therapies: Some families may initially focus their efforts exclusively on treating their child with CAM therapies, which may delay the use of therapies with proven benefits that could have been most helpful for the child.

5. Time trade-off: If the use of CAM therapy involves a significant investment of time and effort, remember that this involves a trade-off. If families spend hours and hours preparing a very restricted diet, it may take away from the time and energy needed to provide other therapies, such as behavioral interventions. Caring for a child with an ASD often involves a great deal of stress, time, and energy, and it is crucial to prioritize efforts towards the most beneficial treatments.
How do I Evaluate The Scientific Evidence on CAM Therapies?

The highest-quality scientific study for evaluating the efficacy of any intervention (CAM or traditional) is the double-blind, placebo-controlled, randomized trial. These studies create groups of patients that are very similar (similar age, similar socioeconomic status, similar severity of disease, etc.) by randomly assigning patients to a treatment or control group. The use of double-blinding indicates that neither the patient nor the persons conducting the study know whether any given patient is receiving the "real" treatment or an identical, inactive (placebo) treatment. Therefore, patients and study personnel should not be influenced when judging whether they have improved (because they do not know whether they are taking the active or the placebo treatment). Once the study is completed, the investigators "unblind" the data, and determine if patients in the active group improved more or less than patients in the placebo group.

Unfortunately, there have been few high quality randomized controlled trials conducted on CAM interventions for ASDs. Therefore, most of the evidence regarding the efficacy of CAM interventions for ASDs comes from anecdotal reports (also known as case reports when they are published in the medical literature). These reports generally describe a child with an ASD who was given a CAM treatment (such as hyperbaric oxygen) and who improved, sometimes dramatically. These reports provide preliminary evidence that a therapy might be effective, but they are extremely limited for several reasons:

1. Case reports have no comparison group, so it is not clear whether similar patients (or the same patient) would have improved without the intervention (this problem is sometimes referred to as a lack of information about the "natural history of the disease"). Because symptoms of ASD often vary from day-to-day or week-to-week in an individual child, it may be difficult to tell if the CAM treatment (or something else) led to the improvement.

2. Case reports are not blinded, so both the patient and the person assessing the outcome are aware of the treatment. This may lead to a biased interpretation of the effect (for example, an acupuncturist might firmly believe in the efficacy of the acupuncture for ASD, and might tend to overestimate the effect). Also, the lack of blinding can lead to a placebo effect, where the observed benefit is not due to the intervention, but to an expectation of benefit.

3. Case reports often involve one or just a few patients, and it is not clear if they are representative of the larger group of children with ASD. 
There are many examples in traditional medicine where widely held medical beliefs (based on case reports or other similar "observational studies") were later proven incorrect by the higher-quality randomized controlled trials. For example, for decades it was well established medical practice that when post-menopausal women were given estrogen, they seemed to have less heart disease. It was not until several large randomized controlled trials were conducted that it was discovered that estrogen had no beneficial effects on heart disease (Hulley et al. 1998).

However, it should also be mentioned that many of the most important scientific discoveries originally came from case reports, and the value of case reports to suggest important possibilities should not be underestimated. For example, the phenomenon of a group of 11 men (who were either homosexual or intravenous drug users) becoming immune deficient was originally described in a case series (Masur et al. 1981). This "anecdotal" observation or case series led to the discovery of the AIDS virus.

For the vast majority of CAM therapies for ASD, there is little or no evidence to document efficacy. However, the lack of evidence should not be equated with a conclusion that a therapy is ineffective. In the absence of scientific evidence, there is an equal chance that any therapy will be beneficial or harmful.

We strongly recommend that you discuss the evidence regarding risks and benefits of each CAM therapy with your child's health care provider to help you decide whether to use and how to monitor the effects of a specific treatment. Additionally, evidence-based reviews are now being conducted for all CAM therapies, and the full scientific reports and plain-language summaries will be made available on-line to help families and clinicians make informed decisions.

\section{References}

Altman, D. G., Schulz, K. F., Moher, D., Egger, M., Davidoff, F., Elbourne, D., et al. (2001). The revised CONSORT statement for reporting randomized trials: Explanation and elaboration. Annals of Internal Medicine, 134, 663-694.

Amminger, G. P., Berger, G. E., Schafer, M. R., Klier, C., Friedrich, M. H., \& Feucht, M. (2007). Omega-3 fatty acids supplementation in children with autism: A double-blind randomized, placebo-controlled pilot study. Biological Psychiatry, 61, 551553. doi:10.1016/j.biopsych.2006.05.007.

Bell, J. G., MacKinlay, E. E., Dick, J. R., MacDonald, D. J., Boyle, R. M., \& Glen, A. C. (2004). Essential fatty acids and phospholipase A2 in autistic spectrum disorders. Prostaglandins, Leukotrienes, and Essential Fatty Acids, 71, 201-204. doi: 10.1016/j.plefa.2004.03.008.

Bu, B., Ashwood, P., Harvey, D., King, I. B., Van de Water, J., \& Jin, L. W. (2006). Fatty acid compositions of red blood cell phospholipids in children with autism. Prostaglandins,
Leukotrienes, and Essential Fatty Acids, 74, 215-221. doi: 10.1016/j.plefa.2006.02.001.

Eisenberg, D. M., Davis, R. B., Ettner, S. L., Appel, S., Wilkey, S., Van Rompay, M., et al. (1998). Trends in alternative medicine use in the United States, 1990-1997: Results of a follow-up national survey. JAMA, 280, 1569-1575.

Eisenberg, D. M., Kessler, R. C., Foster, C., Norlock, F. E., Calkins, D. R., \& Delbanco, T. L. (1993). Unconventional medicine in the United States. Prevalence, costs, and patterns of use. New England Journal of Medicine, 328, 246-252.

FDA announces qualified health claims for omega- 3 fatty acids. (2004). In F. R. P04-89.

Freeman, M. P., Hibbeln, J. R., Wisner, K. L., Davis, J. M., Mischoulon, D., Peet, M., et al. (2006). Omega-3 fatty acids: Evidence basis for treatment and future research in psychiatry. The Journal of Clinical Psychiatry, 67, 1954-1967.

Gilbert, D. L. (2008). Regarding “omega-3 fatty acids supplementation in children with autism: A double-blind randomized, placebo-controlled pilot study”. Biological Psychiatry, 63, e13. doi:10.1016/j.biopsych.2007.03.028.

Green, V. A., Pituch, K. A., Itchon, J., Choi, A., O’Reilly, M., \& Sigafoos, J. (2006). Internet survey of treatments used by parents of children with autism. Research in Developmental Disabilities, 27, 70-84. doi:10.1016/j.ridd.2004.12.002.

Hanson, E., Kalish, L. A., Bunce, E., Curtis, C., McDaniel, S., Ware, J., et al. (2007). Use of complementary and alternative medicine among children diagnosed with autism spectrum disorder. Journal of Autism and Developmental Disorders, 37, 628-636. doi:10.1007/s10803-006-0192-0.

Harrington, J. W., Rosen, L., Garnecho, A., \& Patrick, P. A. (2006). Parental perceptions and use of complementary and alternative medicine practices for children with autistic spectrum disorders in private practice. Journal of Developmental and Behavioral Pediatrics, 27, S156-S161. doi:10.1097/00004703-2006040 02-00014.

Harris, W. S. (2004). Fish oil supplementation: Evidence for health benefits. Cleveland Clinic Journal of Medicine, 71, 208-221.

Hulley, S., Grady, D., Bush, T., Furberg, C., Herrington, D., Riggs, B., et al. (1998). Randomized trial of estrogen plus progestin for secondary prevention of coronary heart disease in postmenopausal women. Heart and Estrogen/progestin Replacement Study (HERS) Research Group. JAMA, 280, 605-613.

Jadad, A. R., Moore, R. A., Carroll, D., Jenkinson, C., Reynolds, D. J., Gavaghan, D. J., et al. (1996). Assessing the quality of reports of randomized clinical trials: Is blinding necessary? Controlled Clinical Trials, 17, 1-12. doi:10.1016/0197-2456(95)00134-4.

Johnson, S. M., \& Hollander, E. (2003). Evidence that eicosapentaenoic acid is effective in treating autism. The Journal of Clinical Psychiatry, 64, 848-849.

Kris-Etherton, P. M., Harris, W. S., \& Appel, L. J. (2002). Fish consumption, fish oil, omega-3 fatty acids, and cardiovascular disease. Circulation, 106, 2747-2757. doi:10.1161/01.CIR.0000 038493.65177.94.

Levy, S. E., Mandell, D. S., Merhar, S., Ittenbach, R. F., \& PintoMartin, J. A. (2003). Use of complementary and alternative medicine among children recently diagnosed with autistic spectrum disorder. Journal of Developmental and Behavioral Pediatrics, 24, 418-423. doi:10.1097/00004703-200312000-00 003.

Masur, H., Michelis, M. A., Greene, J. B., Onorato, I., Stouwe, R. A., Holzman, R. S., et al. (1981). An outbreak of communityacquired Pneumocystis carinii pneumonia: Initial manifestation of cellular immune dysfunction. New England Journal of Medicine, 305, 1431-1438.

Meguid, N. A., Atta, H. M., Gouda, A. S., \& Khalil, R. O. (2008). Role of polyunsaturated fatty acids in the management of 
Egyptian children with autism. Clinical Biochemistry, 41, 10441048. doi:10.1016/j.clinbiochem.2008.05.013.

Patrick, L., \& Salik, R. (2005). The effect of essential fatty acid supplementation on language development and learning skills in autism and asperger's syndrome. Autism Asperger's Digest, JanFeb, pp. 36-37.

Politi, P., Cena, H., Comelli, M., Marrone, G., Allegri, C., Emanuele, E., et al. (2008). Behavioral effects of omega-3 fatty acid supplementation in young adults with severe autism: An open label study. Archives of Medical Research, 39, 682-685. doi: 10.1016/j.arcmed.2008.06.005.

Sawaya, G. F., Guirguis-Blake, J., LeFevre, M., Harris, R., \& Petitti, D. (2007). Update on the methods of the US preventive services task force: Estimating certainty and magnitude of net benefit. Annals of Internal Medicine, 147, 871-875.

Vancassel, S., Durand, G., Barthelemy, C., Lejeune, B., Martineau, J., Guilloteau, D., et al. (2001). Plasma fatty acid levels in autistic children. Prostaglandins, Leukotrienes, and Essential Fatty Acids, 65, 1-7. doi:10.1054/plef.2001.0281.

Wong, H. H., \& Smith, R. G. (2006). Patterns of complementary and alternative medical therapy use in children diagnosed with autism spectrum disorders. Journal of Autism and Developmental Disorders, 36, 901-909. doi:10.1007/s10803-006-0131-0. 